\title{
Effect of zoledronic acid on the doxycycline-induced decrease in tumour burden in a bone metastasis model of human
} breast cancer

\author{
WCM Duivenvoorden ${ }^{1,2}$, S Vukmirović-Popović ${ }^{2}$, M Kalina', E Seidlitz' and G Singh ${ }^{*, 1,2}$ \\ 'Juravinski Cancer Centre, 699 Concession Street, Hamilton, Ontario, Canada L8V 5C2; ${ }^{2}$ Department of Pathology and Molecular Medicine, McMaster \\ University, 1280 Main Street W, Hamilton, Ontario, Canada L8N $3 Z 5$
}

Bone is one of the most frequent sites for metastasis in breast cancer patients often resulting in significant clinical morbidity and mortality. Bisphosphonates are currently the standard of care for breast cancer patients with bone metastasis. We have shown previously that doxycycline, a member of the tetracycline family of antibiotics, reduces total tumour burden in an experimental bone metastasis mouse model of human breast cancer. In this study, we combined doxycycline treatment together with zoledronic acid, the most potent bisphosphonate. Drug administration started 3 days before the injection of the MDA-MB-23I cells. When mice were administered zoledronic acid alone, the total tumour burden decreased by $43 \%$ compared to placebo treatment. Administration of a combination of zoledronic acid and doxycycline resulted in a $74 \%$ decrease in total tumour burden compared to untreated mice. In doxycycline- and zoledronate-treated mice bone formation was significantly enhanced as determined by increased numbers of osteoblasts, osteoid surface and volume, whereas a decrease in bone resorption was also observed. Doxycycline greatly reduced tumour burden and could also compensate for the increased bone resorption. The addition of zoledronate to the regimen further decreased tumour burden, caused an extensive decrease in bone-associated soft tissue tumour burden (93\%), and sustained the bone volume, which could result in a smaller fracture risk. Treatment with zoledronic acid in combination with doxycycline may be very beneficial for breast cancer patients at risk for osteolytic bone metastasis.

British Journal of Cancer (2007) 96, I526- I531. doi:10.1038/sj.bjc.6603740 www.bjcancer.com

Published online 17 April 2007

(c) 2007 Cancer Research UK

Keywords: bone metastasis; breast cancer; tetracyclines; bisphosphonates

Bone is one of the most frequent sites for metastasis of breast and prostate cancer. Bone metastases are often associated, especially in breast cancer, with extensive osteolysis of mineralised collagenous bone matrix (Kanis, 1995) and subsequent hypercalcaemia resulting in significant clinical morbidity. Currently, there is no adequate curative regimen for patients with bone metastasis. The existing standard of care for the treatment of breast cancer-related bone metastasis is the bisphosphonate family of drugs that effectively inhibit the cancer-associated resorption. They are also used as therapeutic agents in clinical disorders characterised by increased osteolysis, such as osteoporosis (Berenson et al, 2001). Unfortunately, treatment with bisphosphonates is not curative, but randomised control trials have shown that zoledronate, the most potent bisphosphonate, decreases the frequency of skeletal-related events, delays the first occurrence of such an event, and reduces pain in bone-metastatic breast cancer patients (Rosen et al, 2004; Kohno et al, 2005). During the 2-year daily administration of oral

\footnotetext{
*Correspondence: Dr G Singh, Juravinski Cancer Centre, 699 Concession Street, Hamilton, Ontario, Canada L8V 5C2;

E-mail: gurmit.singh@hrcc.on.ca

Revised 16 March 2007; accepted 19 March 2007; published online 17 April 2007
}

clodronate, one of the earlier bisphosphonates, to patients with operable breast cancer without evidence of metastatic disease the development of bone metastases is significantly reduced (Powles et al, 2002). Therefore, preclinical evidence that zoledronate may also be used to prevent the onset of bone metastasis would be very worthwhile.

We have previously shown that doxycycline reduces the tumour burden from breast cancer metastasis in nude mice substantially (Duivenvoorden et al, 2002). Doxycycline has several properties relevant for treatment of bone metastasis, as doxycycline can inhibit tumour cell proliferation (Van den Bogert et al, 1983; Fife and Sledge, 1995; Duivenvoorden et al, 1997) and MMP activity, and accumulates at high concentrations in bone (Golub et al, 1991). We have also shown evidence to support the concept that the reduction in tumour burden by doxycycline is likely due to its properties as an inhibitor of tumour cell proliferation and that doxycycline significantly increases several parameters of bone formation (Duivenvoorden et al, 2002).

Bisphosphonates are stable pyrophosphate analogues that also accumulate in bone and very effectively inhibit osteoclastmediated bone resorption. The most potent bisphosphonate is zoledronate, which induces apoptosis of the osteoclast and interferes with the operation of the ruffled border (Green, 2004). In the present study, we combined doxycycline together with zoledronic acid as a possible modality to prevent or reduce the 
tumour burden from bone metastasis using the experimental breast cancer MDA-MB-231 mouse model.

\section{MATERIALS AND METHODS}

\section{Cell line}

The human breast adenocarcinoma MDA-MB-231 cell line obtained from the American Type Culture Collection (Manassas, VA, USA) was maintained in Dulbecco's minimal essential medium supplemented with $10 \%$ fetal bovine serum and antibiotics $\left(100 \mathrm{U} \mathrm{ml}^{-1}\right.$ penicillin sodium, $100 \mu \mathrm{g} \mathrm{ml}^{-1}$ streptomycin sulphate and $0.25 \mu \mathrm{g} \mathrm{ml}^{-1}$ amphotericin B; Invitrogen Canada Inc., Burlington, Ontario, Canada).

\section{Animals}

All protocols for animal studies were reviewed and approved by the Animal Research Ethics Board of McMaster University (Hamilton, Ontario, Canada). Per treatment group 10 female inbred nude (Balb/c nu/nu) mice (Charles River, St. Constant, Quebec, Canada) 5 weeks of age (15-20g) were used. Control animals, not injected with tumour cells, were also included. Intracardiac injections of MDA-MB-231 cells were done according to Arguello et al (1988). Mice were anaesthetised by isoflurane inhalation and the cells $(0.1 \mathrm{ml}$ of cell suspension containing $1 \times 10^{5}$ cells) were injected into the left ventricle of the heart using a 26-gauge needle inserted percutaneously near the midline. Twenty-eight days after injection of the cells, high-resolution radiographic scans of all mice in the prone and lateral position were taken under inhaled isoflurane anaesthesia with a Faxitron X-ray system MX-20 (Faxitron X-ray Corporation, Wheeling, IL, USA). The animals were killed and both tibiae, femora, and humeri, and the spinal column were dissected, fixed in formalin, decalcified, using Decalcifier I (Surgipath, Winnipeg, Manitoba, Canada) and embedded in paraffin.

\section{Treatment}

Doxycycline-containing pellets $(10 \mathrm{mg}$ per pellet with a timedrelease of 21 days; Innovative Research of America, Sarasota, FL, USA) were implanted subcutaneaously 3 days before cancer cell injections. Placebo pellets were used in control animals. At the same time, pellets containing $0.25 \mathrm{mg}$ of $17 \beta$-estradiol (21 dayrelease) were implanted in each animal. On the same day, zoledronic acid (Novartis, Dorval, Quebec, Canada) treatment was started, at a dose of $0.2 \mu \mathrm{g}$ per mouse of zoledronic acid (as $2 \mu \mathrm{g} \mathrm{ml}^{-1} \mathrm{PBS}$ ), given as a subcutaneous injection. The zoledronic acid injections were repeated nine times, every 2 days.

\section{Histology and histomorphometry}

Tumour burden in the long bones and the spinal column of each mouse were measured using a stereological technique as described before (Duivenvoorden et al, 2002). Longitudinal sections (thickness $4 \mu \mathrm{m}$ ) were cut through the middle part of the bone and stained with haematoxylin and eosin $(\mathrm{H} \& \mathrm{E})$. The total cumulative tumour area per animal was calculated using a point grid (point area of $0.02292 \mathrm{~mm}^{2}$ ) and expressed as bone tumour burden. Histomorphometric measurements were performed on cancellous area of the left and right femora starting $1 \mathrm{~mm}$ below the epiphyseal growth plate. Measurements were performed using a digital color camera attached to a light microscope and Northern Eclipse image-analysing software (Empix Imaging Inc., Mississauga, Ontario, Canada). The following parameters were measured: volume occupied by bone, and tumour as a fraction of the total tissue volume (BV/TV and TuV/TV (\%), respectively), number of osteoclasts and osteoblasts per length of trabecular bone surface
(N.Oc/BS and N.Ob/BS $\left(\mathrm{mm}^{-1}\right)$, respectively), eroded bone surface, active resorption surface beneath or in contact with osteoclasts, and osteoid surface as a fraction of the trabecular bone surface (ES/BS, Oc.S/BS, and OS/BS (\%), respectively). To detect tartrate-resistant acid phosphatase (TRAP) activity in the osteoclasts, naphthol AS-BI phosphate (Sigma-Aldrich, Oakville, Ontario, Canada) was used according to van de Wijngaert and Burger (1986).

\section{Statistical analysis}

Tumour burden data were tested for differences between means using Student's $t$-tests. Histomorphometric data were tested for differences between medians using a two-tailed Mann-Whitney test. Differences were considered significant at $P<0.05$.

\section{RESULTS}

Several bisphosphonates have been used in preclinical models of breast cancer bone metastasis. Treatment of mice with ibandronate (Hiraga et al, 2001), risedronate (Sasaki et al, 1995) or zoledronate (Green et al, 2000) results in a reduction of 35,50 and $80 \%$ in osteolytic lesion area as determined by X-ray autoradiography, respectively. Previously, we have shown that doxycycline also significantly reduces tumour burden in the MDA-MB-231 breast cancer bone metastasis model (Duivenvoorden et al, 2002). In this study, we describe the effect of zoledronic acid on the skeletal tumour burden in mice concurrently treated with doxycycline.

Doxycycline was administered to Balb/c nu/nu mice as described previously (Duivenvoorden et al, 2002), resulting in a delivered dose of approximately $15 \mathrm{mg} \mathrm{kg}^{-1}$ day $^{-1}$ starting 3 days before MDA-MB-231 cell injections. Zoledronate was administered at $0.2 \mu \mathrm{g}$ per mouse every 2 days starting at the same time as the doxycycline treatment. We did not observe any adverse effects of any treatment protocol in the mice over the study period of 28 days. Six long bones (both femora, tibiae and humeri), and each vertebral body of the spinal column of each mouse were screened for the microscopical presence or absence of tumour. Previously, it has been shown that the majority of the tumour burden occurs in these bones (Sasaki et al, 1995; Duivenvoorden et al, 2002). Occasionally, the maxillae and pelvic bone show presence of tumour.

Doxycycline alone and in combination with zoledronate significantly reduced the tumour burden. Figure 1A shows the total tumour burden as determined in the femora and spine. These two bones contributed the most to the total tumour burden per animal (Figure 1B). In the placebo group the total cumulative tumour burden (for spine, femora, tibiae and humeri) per animal amounted to $4.59 \pm 1.34 \mathrm{~mm}^{2}$ in tumour-bearing animals $(n=15)$, whereas the total tumour burden in the doxycycline group $(n=12)$ was $2.28 \pm 0.83 \mathrm{~mm}^{2}$, a significant reduction of $50 \%$. In the group of animals that received zoledronate alone $(n=10)$ the tumour burden was $3.62 \pm 1.05 \mathrm{~mm}^{2}$, a number that decreased to $2.16 \pm 0.74 \mathrm{~mm}^{2}$ when doxycycline was added to the treatment protocol $(n=6)$. The tumour burden in the latter group was also significantly decreased when compared to the placebo-treated animals. Interestingly, zoledronic acid effected primarily a reduction in bone-associated soft tissue tumour burden, whereas doxycycline decreased both the bone- and associated soft-tissue tumour burden. Zoledronate caused a $93 \%$ reduction in soft-tissue tumour burden, irrespective of doxycycline treatment, whereas the total tumour burden was merely reduced by 74 and $43 \%$, in the presence or absence of doxycycline, respectively. The combination treatment decreased the tumour burden of both bone and associated soft tissue to a larger extent than either single treatment alone (Figure 1A), although this trend did not reach statistical significance. The numbers of mice per group ended up dissimilar, even though, per repeated experiment, five mice per group were 
injected. The numbers also excluded mice that died very soon after tumour cell injection and ones that did not develop tumours. Moreover, the control and doxycycline-treated mice included mice from previous experiments (Duivenvoorden et al, 2002).

Bone histomorphometry showed that both doxycycline and zoledronate significantly increased several parameters of bone formation in the femora, including osteoid volume (data not shown), osteoid surface and the number of osteoblasts per bone surface (see Table 1, Figure 2B and D). When zoledronic acid alone
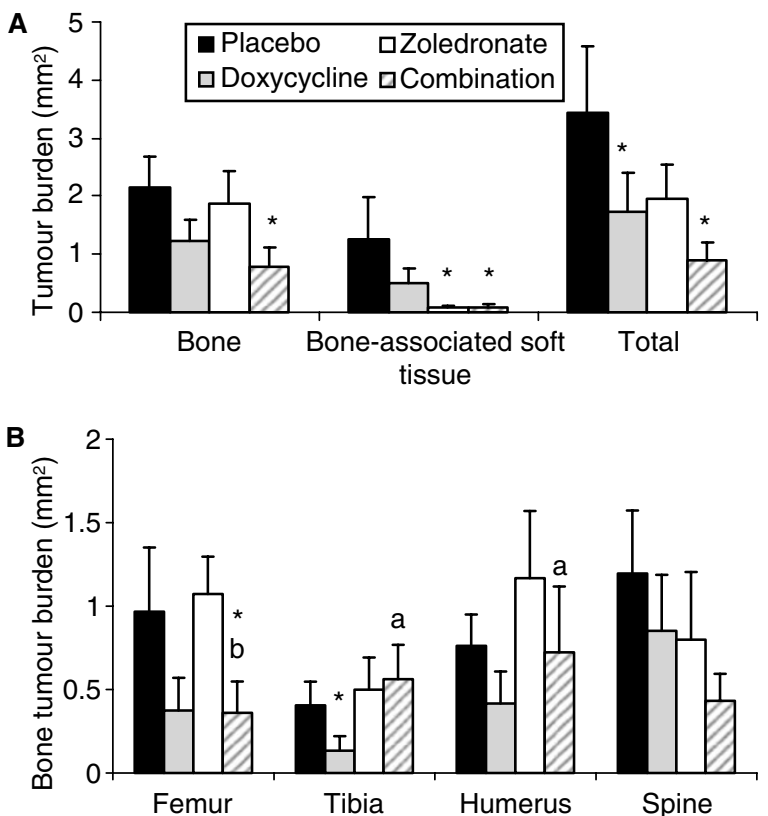

Figure I Effect on tumour burden in a mouse bone metastasis model of human breast cancer (MDA-MB-23I). Mice received a 21-day timedrelease pellets containing $10 \mathrm{mg}$ doxycycline 3 days before intracardiac MDA-MB-23I injections. Other groups of mice received either zoledronic acid (at $0.2 \mu$ g per mouse s.c.) every 2 days alone or in combination with doxycycline. A placebo group was also included. Mice were killed 28 days after cell injection. Longitudinal sections of the spine and femora of tumourbearing animals ( $n=15$ in placebo group, $n=12$ in doxycycline-group, $n=10$ in zoledronate-group, $n=6$ in combination group,) were analysed to determine (A) tumour burden in bone and bone-associated soft tissue tumour burden and (B) average tumour bone burden per evaluated bone (spine, humeri, femora and tibiae). Data represent means \pm s.e. of the combined values of all animals in each of the groups in at least two separate experiments. * Significantly different from corresponding placebo group $(P<0.05)$. ${ }^{a}$ Significantly different from corresponding doxycycline-alone

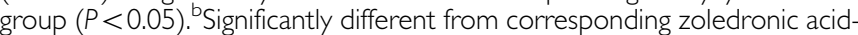
alone group $(P<0.05)$. was used, there was a dramatic and significant increase in bone volume in control animals, which was maintained in the tumourbearing animals (Table 1). The addition of doxycycline to the regimen of zoledronic acid sustained the increase in bone volume and also induced a concomitant decrease in tumour volume even though the difference did not reach significance.

The presence of breast cancer has a profound effect on bone. Skeletal MDA-MB-231 tumours showed extensive marrow colonisation, significant osteolysis, and cortical perforation. Whereas in control animals, hardly any osteoclasts could be found, the number of osteoclasts and the eroded bone surface increased markedly in tumour-bearing bones (Figure 2A and C). In contrast, the structure of the trabecular bone in mice treated with zoledronic acid alone and in combination with doxycycline (Figure 3D and E) seemed better preserved, even in the presence of tumour cells. The mice treated with doxycycline in the presence or absence of zoledronic acid both showed a significant increase in the osteoid surface (Figure 2B); however, this effect could not be maintained in the presence of tumour. Haematoxylin and eosin sections of the femur (Figure 3) clearly show the osteolysis induced by the presence of tumour. Sections stained for TRAP show the increased presence of osteoclastic activity at the tumour-bone interface (Figure 4). In the presence of tumour, we observed noticeable osteolysis on H\&E-stained sections and increased osteoclastic activity at the tumour - bone interface after TRAP staining. We also noticed less TRAP-positive osteoclasts present on the bone surface in both zoledronic acid groups, in the presence or absence of doxycycline (Figure 2C).

A major feature of breast cancer bone metastasis is the uncoupling of bone remodeling. We also observed this phenomenon in tumour-bearing bones and show that doxycycline and zoledronic acid can improve this to some level by increasing bone formation. In doxycycline-treated tumour-bearing mice, the tissue volume occupied by bone was significantly increased, $29.1 \%$ compared to $22.7 \%$ in placebo (Table 1 ). The bone volume further increased to 32.8 and $38.7 \%$ in animals treated with zoledronic acid alone and in combination with doxycycline, respectively. These represented levels of bone volume that were higher than in control mice. This was accompanied by an increase in bone formation parameters (number of osteoblasts (Figure 2D) and osteoid surface (Figure 2B) and volume (Table 1)) and a concomitant decrease in bone resorption parameters, such as number of osteoclasts (Figure 2C) and eroded bone surface (Figure 2A). Interestingly, in control mice, zoledronic acid alone resulted in an increase in the number of both osteoclasts and osteoblasts, suggesting an overall increase in bone remodeling, even in the absence of tumour cells. Moreover, in autoradiographs of mice treated with zoledronate, we also observed dense bone nodules at the sites of rib articulation (data not shown). The aberrant bone formation was noted in zoledronate-treated animals

Table I Effect of treatment with doxycycline or zoledronate alone or combined on bone and tumour volume in mouse bone metastasis model of human breast cancer (MDA-MB-23I) as measured by bone histomorphometry on longitudinal sections of the femora

Bone volume BV/TV (\%)

\begin{tabular}{lcccc}
\cline { 2 - 4 } & Placebo & Doxycycline & Zoledronic acid & Doxycycline and zoledronic acid \\
\hline Control animals & $29.5 \pm 0.32$ & $29.2 \pm 0.97 * *$ & $37.0 \pm 1.79 * *$ & $37.6 \pm 1.84 * * a$ \\
Tumour-bearing animals & $22.7 \pm 1.40 *$ & $29.1 \pm 2.69 * *$ & $32.8 \pm 1.03 * *$ & $38.7 \pm 5.15$
\end{tabular}

Tumour volume TuV/TV (\%)

Tumour-bearing animals

$31.6 \pm 3.68$

$26.4 \pm 6.38$

$28.3 \pm 3.64$

$15.0 \pm 4.97^{a}$

Data represent means \pm s.e. of the combined values of all animals in each of the groups in at least two separate experiments. *Significantly different from corresponding contro (no tumour) group $(P<0.05)$. **Significantly different from corresponding placebo group $(P<0.05)$. ${ }^{\text {a }}$ Significantly different from corresponding doxycycline-alone group $(P<0.05)$. 

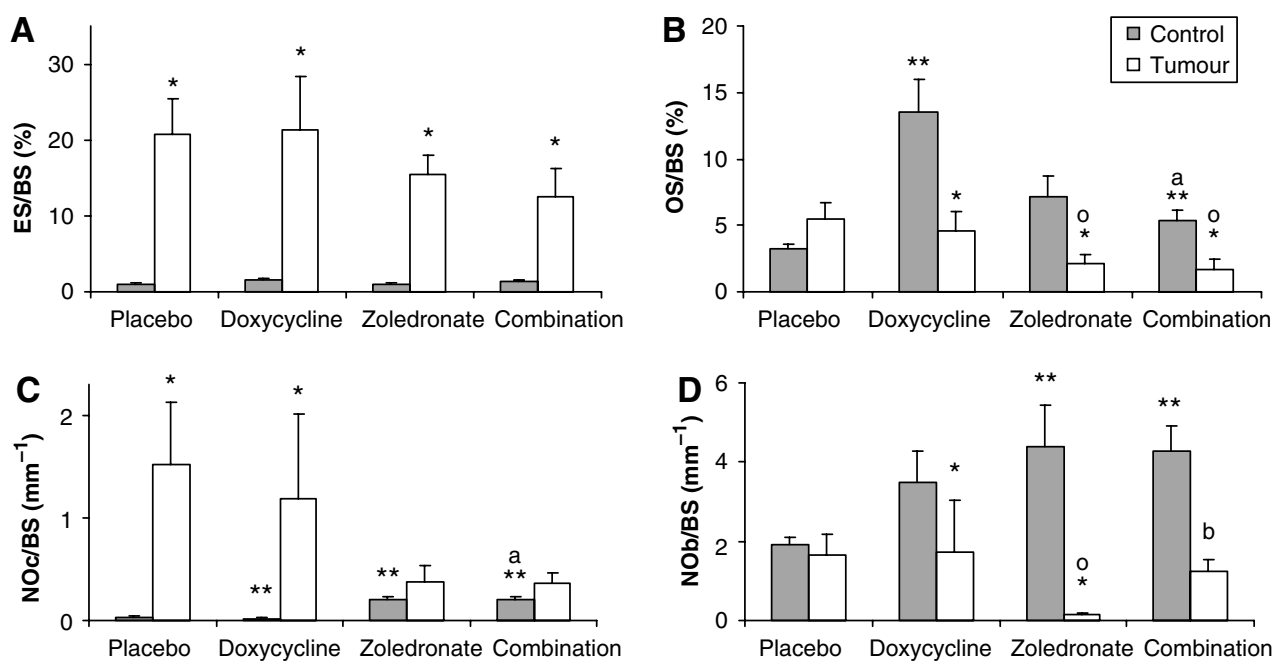

Figure 2 Effect of doxycycline treatment on bone histomorphometric parameters in a mouse bone metastasis model of human breast cancer (MDA-MB23 I). Longitudinal sections of the femora were analysed to determine $(\mathbf{A})$ eroded bone surface as a fraction of the trabecular bone surface (\%), and (B) osteoid surface as a fraction of the trabecular bone surface (\%), (C) number of osteoclasts per mm trabecular bone surface, and (D) number of osteoblasts per $\mathrm{mm}$ of trabecular bone surface. Data represent means \pm s.e. of the combined values of all animals in each of the groups in at least two separate experiments $(n \geqslant 3)$. * Significantly different from corresponding control group (no tumour cells injected) $(P<0.05)$. *** Significantly different from placebo control animals (no tumour cells injected) $(P<0.05)$. ${ }^{\circ}$ Significantly different from placebo tumour-bearing animals $(P<0.05)$. ${ }^{a}$ Significantly different from corresponding doxycycline-alone group $(P<0.05)$. 'Significantly different from corresponding zoledronic acid-alone group $(P<0.05)$.
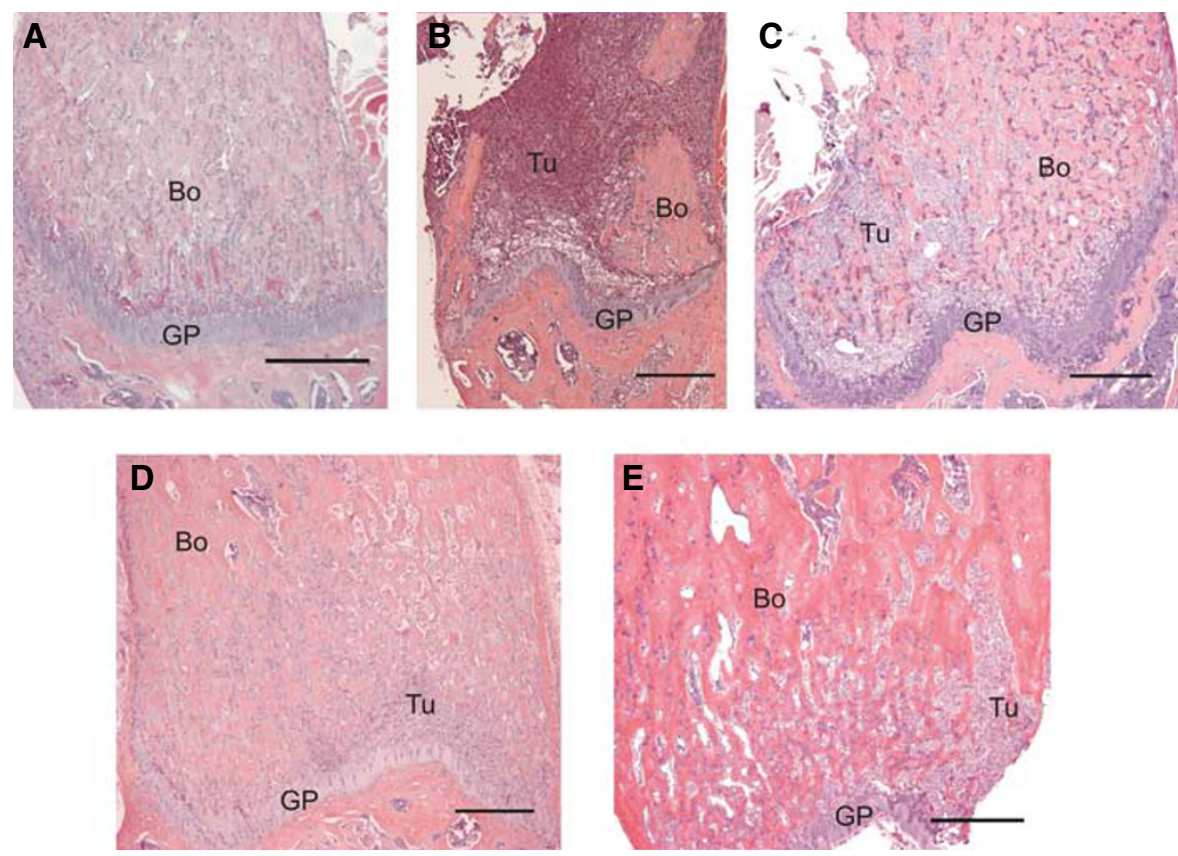

Figure 3 (A) Histological appearance of the left femur of a control mouse (untreated, no cell injections) stained with H\&E. (B-E) Histological appearance of skeletal tumours in the left femora of mice injected with MDA-MB-23I cells after H\&E staining. Mice were treated with (B) placebo, (C) doxycycline, (D) zoledronic acid, or (E) with both doxycycline and zoledronic acid. (Original magnification $\times 100$, Bar $=150 \mu \mathrm{m}$ ). Bo, bone; GP, growth plate; Tu, tumour.

irrespective of doxycycline treatment or the presence of tumour cells.

\section{DISCUSSION}

Using the same bone metastasis model, zoledronate has been shown to markedly reduce the area of osteolytic lesions as detected by X-ray autoradiography, independent of time of administration (concurrently with the tumour cell injections or after the establishment of bone metastasis) (Peyruchaud et al, 2001) and of the dose administered (0.2, 1 and $5 \mu \mathrm{g}$ per mouse; Green et al, 2000). When GFP-labelled MDA-MB-231 cells are used, the effect of zoledronate on the fluorescence as a measure of the amount of tumour cells is less pronounced than on the osteolytic lesion area (Peyruchaud et al, 2001). Similarly, treatment with olpadronate, another bisphosphonate, demonstrates that the tumour burden in the initial phases is more heavily impacted and returns to vehicle- 

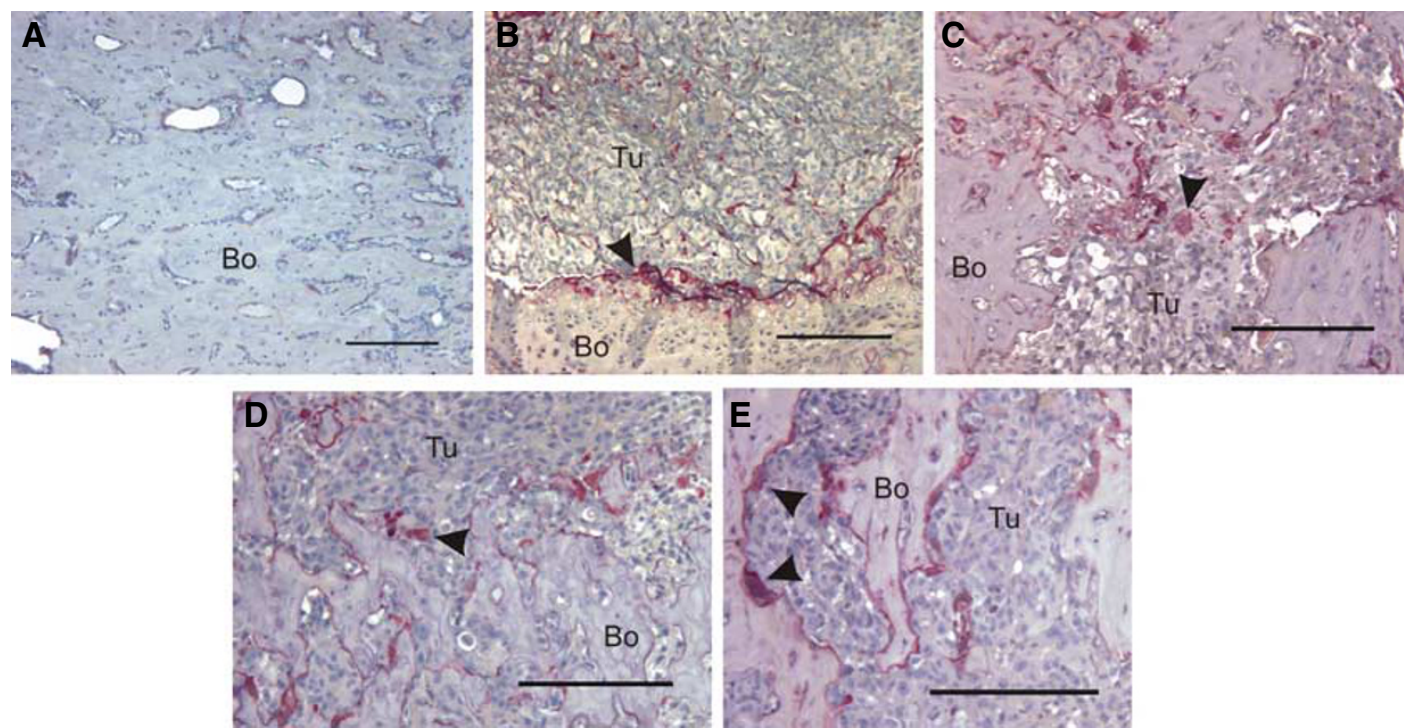

Figure 4 (A) Histological appearance of the left femur of a control mouse (untreated, no cell injections) stained for TRAP. No osteoclasts were observed. (B-E) Histological appearance of skeletal tumours in the left femora of mice injected with MDA-MB-23I cells showing TRAP-positive osteoclasts (arrowheads). Mice were treated with (B) placebo, (C) doxycycline, (D) zoledronic acid or (E) with both doxycycline and zoledronic acid. (Original magnification $\times 400$, Bar $=20 \mu \mathrm{m})$. Bo, bone; Tu, tumour; arrowheads, osteoclasts.

treated levels by the end of the 47-day treatment. During the same time frame, radiography shows a sustained and extensive decrease in osteolytic lesions (van der Pluijm et al, 2005), thus substantiating the primary mechanism of action of bisphosphonates as anti-resorptive. We observed primarily a large reduction in boneassociated soft tissue tumour burden $(93 \%)$ after administration of zoledronate. This is also supported by data using a $4 \mathrm{~T} 1 /$ luc mouse breast cancer model showing that zoledronic acid results in an inhibition of visceral metastasis (Hiraga et al, 2004) and by Corey et al (2003), who provided evidence to suggest that the inhibitory effects of zoledronate may not only be associated to its effect on osteolysis, but also on cancer cell proliferation and apoptosis.

When comparing both compounds, our results showed that doxycycline induced a larger decrease in tumour burden, whereas zoledronate was more capable of sustaining the bone volume, in spite of an increase in bone resorption. Similar results were reported using osteoblastic LuCaP23.1 or osteolytic PC-3 prostate cancer cells injected into mouse tibiae. Zoledronic acid treatment also induces a decrease in tumour volume and a concomitant increase in bone volume (Corey et al, 2003). When we administered both drugs the result was a substantial decrease in tumour burden and a sustained bone volume. Future investigations will focus on potential effects on local concentrations of each of the osteotropic drugs in the bone and on the specific targets of both drugs. The main target of zoledronic acid appears to be the induction of apoptosis (Corey et al, 2003; Quinn et al, 2005), whereas doxycycline induces a G1 cell cycle arrest (Van den Bogert et al, 1986). Hiraga et al (2001) describe a substantial increase in apoptotic MDA-MB-231 cells in the bones of mice treated with ibandronate, especially at the bone-tumour interface. Several other mechanisms of action have also been suggested for both drugs. In vitro, zoledronate has also been reported to stimulate osteoprotegerin production in primary human osteoblasts (Viereck et al, 2002), inhibit prostate cancer cell proliferation (Corey et al, 2003), MMP activity (Boissier et al, 2000) and adhesion to bone extracellular matrix (Boissier et al, 1997).

Whereas the current study clearly demonstrates the benefit of combined treatment when both drugs are administered simultaneously starting 3 days before cell injections, future experiments will include sequential administration, and starting several weeks after the cell injections. This will help to determine whether doxycycline could also be beneficial for patients with already established bone metastasis and who are currently receiving zoledronic acid and vice versa. In conclusion, doxycycline greatly reduced tumour burden and could also compensate for the increased bone resorption frequently associated with bone metastasis from breast cancer. The addition of zoledronate to the treatment regimen further decreased tumour burden and effected an extensive decrease in bone-associated soft tissue tumour burden, independent of doxycycline treatment. Moreover, the combination also sustained bone volume, which could greatly reduce the risk of fracture. Treatment with zoledronic acid in combination with doxycycline may be very beneficial for breast cancer patients at risk for osteolytic bone metastasis.

\section{ACKNOWLEDGEMENTS}

We thank the Canadian Breast Cancer Research Initiative and Canadian Institutes of Health Research for financial support. We are indebted to Scott Su and Dr S Lhoták for excellent technical assistance.

\section{REFERENCES}

Arguello F, Bages RB, Frantz CN (1988) A murine model of experimental metastasis in bone and bone marrow. Cancer Res 48: 6876-6881

Berenson JR, Rosen LS, Howell A, Porter L, Coleman RE, Morley W, Dreicer

R, Kuross SA, Lipton A, Seaman JJ (2001) Zoledronic acid reduces skeletal-related events in patients with osteolytic metastases. Cancer 91: $1191-1200$

Boissier S, Ferreras M, Peyruchaud O, Magnetto S, Ebetino FH, Colombel M, Delmas P, Delaisse JM, Clezardin P (2000) Bisphosphonates inhibit 
breast and prostate carcinoma cell invasion, an early event in the formation of bone metastases. Cancer Res 60: 2949-2954

Boissier S, Magnetto S, Frappart L, Cuzin B, Ebetino FH, Delmas PD, Clezardin P (1997) Bisphosphonates inhibit prostate and breast cancer carcinoma cell adhesion to unmineralized and mineralized bone extracellular matrices. Cancer Res 57: 3890-3894

Corey E, Brown LG, Quinn JE, Poot M, Roudier MP, Higano CS, Vessella RL (2003) Zoledronic acid exhibits inhibitory effects on osteoblastic and osteolytic metastases of prostate cancer. Clin Cancer Res 9: 295-306

Duivenvoorden WCM, Hirte HW, Singh G (1997) Use of tetracycline as an inhibitor of matrix metalloproteinase activity secreted by human bonemetastasizing cancer cells. Invasion Metastasis 17: 312-322

Duivenvoorden WCM, Vukmirovic-Popovic S, Lhotak S, Seidlitz E, Hirte HW, Tozer RG, Singh G (2002) Doxycycline decreases tumor burden in a bone metastasis model of human breast cancer. Cancer Res 62: $1588-1591$

Fife RS, Sledge Jr GW (1995) Effects of doxycycline on in vitro growth, migration, and gelatinase activity of breast carcinoma cells. J Lab Clin Med 125: $407-411$

Golub LM, Ramamurthy NS, McNamara TF, Greenwald RA, Rifkin BR (1991) Tetracyclines inhibit connective tissue breakdown: new therapeutic implications for an old family of drugs. CRC Crit Rev Oral Biol Med 2: $297-321$

Green JR (2004) Bisphosphonates: preclinical review. Oncologist 9: 3-13

Green JR, Gschaidmeier H, Yoneda T (2000) Zoledronic acid potently inhibits tumour-induced osteolysis in two models of breast cancer metastasis to bone. Ann Oncol 11: 14

Hiraga T, Williams PJ, Mundy GR, Yoneda T (2001) The bisphosphonate Ibandronate promotes apoptosis in MDA-MB-231 human breast cancer cells in bone metastases. Cancer Res 61: 4418-4424

Hiraga T, Williams PJ, Ueda A, Tamura D, Yoneda T (2004) Zoledronic acid inhibits visceral metastases in the $4 \mathrm{~T} 1 /$ luc mouse breast cancer model. Clin Cancer Res 10: 4559-4567

Kanis JA (1995) Bone and cancer: pathophysiology and treatment of metastases. Bone 17: 101S-105S

Kohno N, Aogi K, Minami H, Nakamura S, Asaga T, Iino Y, Watanabe T, Goessl C, Ohashi Y, Takashima S (2005) Zoledronic acid significantly reduces skeletal complications compared with placebo in Japanese women with bone metastases from breast cancer: a randomized, placebocontrolled trial. J Clin Oncol 23: 3314-3321
Peyruchaud O, Winding B, Pecheur I, Serre CM, Delmas P, Clezardin P (2001) Early detection of bone metastases in a murine model using fluorescent human breast cancer cells: application to the use of the bisphosphonate zoledronic acid in the treatment of osteolytic lesions. J Bone Miner Res 16: $2027-2034$

Powles T, Paterson S, Kanis JA, McCloskey E, Ashley S, Tidy A, Rosenqvist K, Smith I, Ottestad L, Legault S, Pajunen M, Nevantaus A, Mannisto E, Suovuori A, Atula S, Nevalainen J, Pylkkanen L (2002) Randomized, placebo-controlled trial of clodronate in patients with primary operable breast cancer. J Clin Oncol 20: 3219-3224

Quinn JE, Brown LG, Zhang J, Keller ET, Vessella RL, Corey E (2005) Comparison of Fc-osteoprotegerin and zoledronic acid activities suggests that zoledronic acid inhibits prostate cancer in bone by indirect mechanisms. Prostate Cancer Prostatic Dis 8: 253-259

Rosen LS, Gordon DH, Dugan Jr W, Major P, Eisenberg PD, Provencher L, Kaminski M, Simeone J, Seaman J, Chen BL, Coleman RE (2004) Zoledronic acid is superior to pamidronate for the treatment of bone metastases in breast carcinoma patients with at least one osteolytic lesion. Cancer 100: $36-43$

Sasaki A, Boyce BF, Story B, Wright KR, Chapman M, Boyce R, Mundy GR, Yoneda T (1995) Bisphosphonate risedronate reduces metastatic human breast cancer burden in bone in nude mice. Cancer Res 55: 3551-3557 van de Wijngaert FP, Burger EH (1986) Demonstration of tartrate-resistant acid phosphatase in un-decalcified, glycolmethacrylate-embedded mouse bone: a possible marker for (pre)osteoclast identification. J Histochem Cytochem 34: 1317-1323

Van den Bogert C, Dontje BH, Kroon AM (1983) Arrest of in vivo growth of a solid Leydig cell tumor by prolonged inhibition of mitochondrial protein synthesis. Cancer Res 43: 2247-2251

Van den Bogert C, van Kernebeek G, de Leij L, Kroon AM (1986) Inhibition of mitochondrial protein synthesis leads to proliferation arrest in the G1phase of the cell cycle. Cancer Lett 32: 41-51

van der Pluijm G, Que I, Sijmons B, Buijs JT, Lowik CW, Wetterwald A, Thalmann GN, Papapoulos SE, Cecchini MG (2005) Interference with the microenvironmental support impairs the de novo formation of bone metastases in vivo. Cancer Res 65: 7682-7690

Viereck V, Emons G, Lauck V, Frosch KH, Blaschke S, Grundker C, Hofbauer LC (2002) Bisphosphonates pamidronate and zoledronic acid stimulate osteoprotegerin production by primary human osteoblasts. Biochem Biophys Res Commun 291: 680-686 